\title{
Antiviral Therapy
}

\section{Evolution of HIV-1 drug resistance after virological failure of first-line antiretroviral therapy in Lusaka Zambia}

F Parker Hudson, Lloyd Mulenga, Andrew O Westfall, Ranjit Warrier, Aggrey Mweemba, Michael S Saag, Jeffrey SA Stringer, Joseph J Eron, Benjamin H Chi

Antiviral Therapy 2019; 10.3851/IMP3299

Submission date Acceptance date Publication date 5th November 2018

24th February 2019

12th April 2019

This provisional PDF matches the article and figures as they appeared upon acceptance.

Copyedited and fully formatted PDF and full text (HTML) versions will be made available soon.

For information about publishing your article in Antiviral Therapy go to http://www.intmedpress.com/index.cfm?pid=12 
This pdf is for personal use only. To obtain commercial reprints, please contact the editorial office on +442073980700

Publication: Antiviral Therapy; Type: Original article

DOI: $10.3851 /$ IMP3299

\section{Original article}

\section{Evolution of HIV-1 drug resistance after virological failure of first-line antiretroviral therapy in Lusaka Zambia}

F Parker Hudson ${ }^{1 *}$, Lloyd Mulenga ${ }^{2}$, Andrew O Westfall ${ }^{3}$, Ranjit Warrier ${ }^{4}$, Aggrey Mweemba ${ }^{2}$, Michael S Saag ${ }^{5}$, Jeffrey SA Stringer ${ }^{6}$, Joseph J Eron ${ }^{7}$, Benjamin H Chi6

${ }^{1}$ Department of Internal Medicine, University of Texas Austin, Austin, TX, USA

${ }^{2}$ School of Medicine, University of Zambia, Lusaka, Zambia

${ }^{3}$ Department Biostatistics, University of Alabama at Birmingham, Birmingham, AL, USA

${ }^{4}$ Centre for Infectious Disease Research in Zambia, Lusaka, Zambia

${ }^{5}$ Department of Infectious Diseases, University of Alabama at Birmingham, Birmingham, AL, USA

6UNC Institute for Global Health and Infectious Diseases, University of North Carolina at Chapel Hill, Chapel Hill, NC, USA

${ }^{7}$ Department of Infectious Diseases, University of North Carolina at Chapel Hill, Chapel Hill, NC, USA

*Corresponding author e-mail: parker.hudson@austin.utexas.edu

\section{Abstract}

Background: HIV viral load (VL) and resistance testing are limited in sub-Saharan Africa, so individuals may have prolonged time on failing first-line antiretroviral therapy (ART). Our objective was to describe the evolution of drug resistance mutations among adults failing first-line ART in Zambia.

Methods: We analyzed data from a trial of VL monitoring in Lusaka, Zambia. From 2006 to 2011, 12 randomized sites provided either routine VL monitoring (intervention) or discretionary (control) after ART initiation. Samples were collected prospectively following the same schedule in each arm but analyzed retrospectively in the control group. For those with virologic failure (VF) (>400 copies $/ \mathrm{mL}$ ), HIV genotyping was performed retrospectively on baseline (BL) and on all subsequent specimens until censored due to study completion, withdrawal, or death.

Results: Of 1,973 enrollees, 165 (8.4\%) developed VF. 464 genotype results were available including $132(80 \%)$ at BL, $116(70 \%)$ at VF, and $125(76 \%)$ had at least one result between VF and censoring. Major NRTI or NNRTI mutations increased from $26 \%(B L)$ to $82 \%$ (VF) to $89 \%$ at last genotype (LG). M184 mutations increased from $2 \%$ to $59 \%$ to $71 \%$; K65R from $2 \%$ to $11 \%$ to $13 \%$; 2 or more TAMs from $1 \%$ to $3 \%$ to $12 \%$. Among those on a failing TDF-based regimen, TDF resistance increased from $42 \%$ to $58 \%$. 
This pdf is for personal use only. To obtain commercial reprints,

please contact the editorial office on +442073980700

Publication: Antiviral Therapy; Type: Original article

DOI: $10.3851 /$ IMP3299

Conclusions: We found substantial resistance to NRTIs and NNRTIs at VF with incremental increases after VF while still on a failing first-line ART; this resistance may compromise attainment of the UNAIDS 90-90-90 goals.

Accepted 24 February 2019, published online 12 April 2019

Short Title: HIV drug resistance in Zambia

\section{Introduction}

In much of sub-Saharan Africa, access to routine HIV viral load (VL) monitoring and drug resistance testing remains limited [1]. As part of the global strategy to achieve $90 \%$ viral suppression, the World Health Organization recommends routine VL monitoring to detect virologic failure (VF) before clinical symptoms of disease progression and to reduce the accumulation of antiretroviral drug resistance mutations [2]. Drug resistance increases the need for alternative antiretroviral therapy (ART) regimens that may be less effective and more expensive, with less favorable toxicity profiles and greater pill burden. Further, it may limit the effectiveness of pre-exposure prophylaxis and first-line ART if resistant subtypes are transmitted within communities. At a population level, such resistance could threaten the success of rapidly expanding HIV programs in sub-Saharan Africa to achieve the UNAIDS 90-90-90 goals since most first line ART still includes non-nucleoside reverse transcriptase inhibitors (NNRTIs) [3].

With limited testing available in clinical settings, data on HIV drug resistance has been extrapolated from research studies and surveillance data [4]. In the multi-country PASER-M cohort study, for example, NNRTI resistance was high at VF (80\%); the M184V mutation was present in 70\%; K65R was detected in 7 adults (11\%); and tenofovir resistance increased to $30 \%$ with continued VF [5,6]. In the TenoRes study, tenofovir resistance was found in nearly $60 \%$ of adults on tenofovir-based first-line ART at the time of VF [7]. In small study from Uganda, high rates of NNRTI resistance were reported after one $(50 \%, 8 / 16)$, two $(69 \%, 9 / 13)$, and three $(60 \%, 6 / 10)$ years following the initiation of first-line ART [8].

Although these insights on HIV drug resistance in Africa have been valuable, there are limitations to studies thus far. Most have been cross-sectional in design and thus unable to compare resistance to baseline profiles [8,9]. Convenience sampling has limited generalizability. Information about the evolution of resistance mutations over time in sub-type $C$ viruses among participants who remain on a failing ART regimen due to delayed recognition of VF has not been well described. We seek to address these gaps by analyzing data from a large cluster-randomized trial of routine viral load monitoring in Zambia [10,11]. Our objective was to describe the evolution of drug resistance mutations from ART over time among HIVinfected adults failing first-line ART. The trial cohort created a unique opportunity to answer this study question, since samples were collected prospectively at regular intervals while participants remained on a failing first-line regimen, but they were analyzed for drug resistance retrospectively. 
This pdf is for personal use only. To obtain commercial reprints, please contact the editorial office on +442073980700

Publication: Antiviral Therapy; Type: Original article DOI: 10.3851/IMP3299

\section{Methods}

\section{Study design}

Our current study is a secondary analysis of data collected during a cluster-randomized trial of routine viral load monitoring on all-cause mortality and HIV disease progression. The full methods of that trial have been reported elsewhere $[10,11]$. Briefly, we randomized 12 facilities in Lusaka, Zambia to one of two strategies for detecting treatment failure among adults initiating ART. Participants were enrolled between December 2006 and May 2008 and followed for up to 36 months. At the intervention sites, participants received routine viral load monitoring at three months, six months, and every six months thereafter. At the control sites, participants were evaluated for treatment failure using clinical and immunologic criteria per the standard of care recommended by the Zambian Ministry of Health. When these findings were found to be conflicting, "discretionary" viral load testing was used to determine treatment failure at the control sites [12]. All participants had a similar schedule for specimen collection; however, in the control arm, virologic testing was not part of clinical care and was therefore conducted at the end of the study.

Patients were eligible to participate if they met criteria for ART initiation according to the Zambian national guidelines, which at that time required either CD4 count $<350$ or World Health Organization clinical stage 3 or 4 . Exclusion criteria included prior ART exposure except for prevention of mother to child transmission, serious illness requiring hospitalization at time of ART initiation, or co-morbidities that may limit participation in study procedures. Participants were prescribed ART according to the prevailing clinical guidelines from the Zambian Ministry of Health. First-line regimens comprised two NRTIs (zidovudine [ZDV], lamivudine [3TC], emtricitabine [FTC], tenofovir disoproxil fumarate [TDF], stavudine $[\mathrm{D} 4 \mathrm{~T}]$ ) and an NNRTI (nevirapine [NVP] or efavirenz [EFV]) in the following combinations: $Z D V+3 T C+N V P, \quad Z D V+3 T C+E F V, \quad D 4 T+3 T C+N V P, \quad D 4 T+3 T C+E F V, \quad T D F+F T C+N V P, \quad$ and TDF+FTC+EFV. The protocol was approved by the University of Zambia Biomedical Research Ethics Committee (Lusaka, Zambia) and institutional review boards at the University of Alabama at Birmingham (Birmingham, AL, USA) and the University of North Carolina at Chapel Hill (Chapel Hill, NC, USA). The study was registered on clinicaltrials.gov (NCT00929604).

\section{Analysis cohort}

For this secondary analysis, we included only participants who experienced VF, diagnosed by viral load testing either prospectively, over the course of the trial (intervention arm) or retrospectively, using stored specimens at the end of the trial (control arm). VF was defined as the first of two consecutive viral loads greater than 400 copies/mL plasma RNA six months after ART initiation with a buffer of 30 days for tests drawn just before 6 months. HIV-1 viral loads were measured with Roche Amplicor HIV-1 RNA Monitor kit (version 1.5; Roche Molecular Diagnostics, Pleasanton, CA, USA). After VF was identified, HIV genotyping for antiretroviral drug resistance was performed on the baseline (i.e., enrollment) specimen 
This pdf is for personal use only. To obtain commercial reprints, please contact the editorial office on +442073980700

Publication: Antiviral Therapy; Type: Original article DOI: 10.3851/IMP3299

and all subsequent specimens on first-line therapy until the participant changed to second-line therapy or was censored due to study completion, withdrawal, or death. The VF genotype was reported from the first of the two viral loads over 400 copies $/ \mathrm{mL}$ in all but 5 cases; the confirmatory viral load was used for the genotype in these five cases because two were below the genotype viral threshold, one was not stored, and two had technical failure; all were performed within 90 days of the first VF viral load (range: 25-87). The threshold of detection for the genotype assay - set at the time of study design - was a viral load of 1,000 copies/mL HIV-1 RNA, which was extracted, amplified, and sequenced using Sanger sequencing on an Applied Biosystems 3130xl instrument [13]. The sequence fragments were aligned using Sequencher software version 5.0 (Gene Codes Corporation, Ann Arbor, MI). Base calling was conducted automatically with a $25 \%$ threshold for mixed bases. All chromatograms were also inspected visually to ensure base calling accuracy. Fasta sequences were submitted to the Stanford HIV Drug Resistance Database in 2016 to predict drug susceptibilities (version 8.2), and the Stanford susceptibility classifications were reported directly from the program.

\section{Statistical analysis}

NRTI mutations were categorized according to the protocol developed by Tang et al. [14] This approach included 34 surveillance drug-resistance mutations at 15 reverse transcriptase (RT) positions (M41L, K65R, K67NGE, T69D, T69ins, K70RE, L74VI, V75MTAS, F77L, Y115F, F116Y, Q151M, M184VI, L210W, T215YFISCDVE, K219QERN), as well as K65N, T69del, and K70QGT. The thymidine analog mutations (TAMs) were M41L, D67NG, K70R, L210W, T215YF, and K219QE. The NNRTI mutations were analyzed based on the 18 surveillance drug-resistance mutations at 10 RT positions (L100I, K101E, K103NS, V106AM, V179F, Y181CI, Y188LCH, G190ASEQV, P225H, M230L, P236L). Additional mutations were included that have a similar predicted phenotypic effect (L100V, K101PH, V179LD, Y181V, G190V, M230I) or have important implications for future therapy (E138AGKQ). The Pan-NNRTI category for Table 2 includes any key mutations for second generation NNRTI resistance: L100I, K101EP, Y181, Y188L, G190, M230L.

We performed univariate descriptive statistics on the total study population and those experiencing VF. We analyzed mutations at time of VF and cumulatively through the last available genotype on a failing first line regimen; if a mutation was detected at one time point, it was considered present at all subsequent time points. The mutation frequencies were tabulated as a percentage of those participants who experienced VF and had a genotype. Baseline resistance as any NRTI or NNRTI mutation versus none was compared with chi square tests at each month and year of study enrollment and categorized by year quarter to determine if baseline resistance increased over the study. Survival curves of time to VF were created using Kaplan-Meier analysis comparing any baseline NRTI or NNRTI mutation to none. All analyses were performed with SAS version 9.4 (SAS Institute, Cary, NC). 
This pdf is for personal use only. To obtain commercial reprints,

please contact the editorial office on +442073980700

Publication: Antiviral Therapy; Type: Original article

DOI: 10.3851/IMP3299

\section{Role of the funding source}

The funders had no role in the design, analysis, or writing of this article; however, representatives from the US Centers of Disease Control and Prevention reviewed a final draft of the manuscript prior to submission.

\section{Results}

Of 1,973 participants enrolled in the parent trial, 165 (8.4\%) developed VF over 4,446 person years of study follow-up. Participants who developed VF had a median age of 32.1 years and $60 \%$ were women. Subtype C was the predominant HIV-1 virus (93\%), and 57\% had a baseline plasma HIV viral load greater than 100,000 copies/mL. In the VF group, $63 \%$ had WHO stage III or IV disease at enrollment; the median CD4 count was 126 cells $/ \mathrm{mm}^{3}$ (interquartile range [IQR]: 57-182). 84\% started an NVP-based regimen, while the remaining $16 \%$ started an EFV-based regimen. The NRTI backbones included ZDV (51\%), d4T (32\%), and TDF (16\%). In Table 1, we compared baseline characteristics of our analysis cohort (i.e., those with VF) to all trial participants. When stratified by NRTI backbone, $10 \%$ on ZDV had $V F$, compared to $8 \%$ on $d 4 T$ and $6 \%$ on TDF $(p=0.15)$. When stratified by NNRTI, $9 \%$ of those on NVP had VF compared to $5 \%$ with EFV ( $p=0.02)$.

Among the 165 participants with VF, 464 HIV genotype results were available, including 132 (80\%) at baseline and $116(70 \%)$ at time of VF. 125 (76\%) participants had at least one genotype between VF and time of censoring, which was defined as a switch to second-line therapy, death, withdrawal from the study, or completion of study follow-up. The median time from ART initiation (i.e., baseline) to the VF genotype was 356 days (IQR: 175-553). The median time from VF genotype to last available HIV genotype, prior to the switch to a second-line regimen, was 202 days (IQR: 139-461). Thus our analysis cohort represents a cumulative 191 person-years on a failing first-line ART regimen. Reasons for missing genotype results included: viral load below the threshold for HIV genotyping, unavailable sample from the visit, or laboratory technical failure (Figure 1).

Of the 132 participants with HIV genotype results at ART initiation, 36 (27\%) had a major NRTI or NNRTI resistance mutation (Table 2). This included 30 (23\%) with at least one NNRTI mutation, including K103N ( $n=13,10 \%)$ and pan-NNRTI resistance $(n=12,9 \%)$. In addition, 13 (10\%) had an NRTI mutation at baseline, including M184 $(n=3,2 \%)$ and K65R $(n=2,2 \%)$. Presence of NNRTI resistance at ART initiation did not affect the time to VF (Figure 2). Baseline resistance did not change over the course of study enrollments $(p=0.6)$. Women who reported using PMTCT services in the past had twice the baseline rate of NNRTI resistance (42\%) compared to men (20\%) and women not reporting prior PMTCT exposure (16\%).

We examined results from both the HIV genotype results at VF $(n=116 / 165,70 \%)$ and the last available genotype (LG) ( $n=151 / 165,92 \%)$. At VF, 81\% (94/116) had a major NRTI or NNRTI mutation, 
This pdf is for personal use only. To obtain commercial reprints, please contact the editorial office on +442073980700

Publication: Antiviral Therapy; Type: Original article DOI: 10.3851/IMP3299

which increased to $89 \%$ (134/151) (Table 2). M184 mutations increased from 2\% at baseline (BL) to 59\% at VF to $71 \%$ at LG; $12 \%$ of participants had 2 or more TAMs. $15 \%$ of those with VF on TDF or ZDV developed TAMs, compared to $6 \%$ on participants on $d 4 T$ ( $p=0.14)$. K65R mutations increased from $2 \%$ (BL) to $11 \%$ (VF) to $13 \%$ (LG); K65R mutations arose in $46 \%(12 / 26)$ on TDF, $10 \%(5 / 48)$ on d4T, and $4 \%$ (3/76) on AZT. At VF, 81\% had at least one major NNRTI mutation and 52\% had a pan-NNRTI mutation. Pan-NNRTI mutations were highly prevalent in participants taking NVP (83/89; 93\%). The viral load monitoring arm had less accumulation of TAMs compared to the control arm in the last genotype (7\% v 16\%; Supplement Table 2a). Prolonged failure over 9 months on a first line regimen increased mutation frequency for M184V, TAMs, and pan-NNRTI mutations (Supplement Table 2b); however the majority of mutations had already accumulated by the time virologic failure was detected.

Predicted drug susceptibilities were greatly reduced at VF and further with the last available genotype (Figure 3). FTC/3TC susceptibility decreased from $98 \%$ at baseline to $39 \%$ at VF to $26 \%$ at LG. AZT susceptibility decreased from $98 \%$ (BL) to $91 \%$ (VF) to $77 \%$ (LG). TDF decreased from $98 \%$ (BL) to $87 \%$ (VF) to $77 \%$ (LG); among those on a failing TDF-based regimen, TDF resistance increased from $42 \%(8 / 19, V F)$ to $58 \%(15 / 26, L G) .70 \%$ developed resistance to second generation NNRTIs. Rilpivirine susceptibility or potential low-level resistance decreased from $84 \%$ (BL) to $38 \%(V F)$ to $30 \%(L G)$. High level resistance increased from $3 \%$ (BL) to $29 \%$ (VF) to $43 \%$ (LG). Etravirine susceptibility or potential low-level resistance decreased from $91 \%(\mathrm{BL})$ to $53 \%$ (VF) to $42 \%$ (LG); likewise the high-level resistance increased from $0 \%(\mathrm{BL})$ to $4 \%$ (VF) to $10 \%$ (LG). Except for the accumulation of TAMs leading to increased ZDV resistance, there was no difference in stratified analysis of resistance by the control versus intervention arm of the parent cluster randomized trial (supplemental figure).

\section{Discussion}

In this study of adult patients initiating NNRTI-based ART in Zambia, over 90\% of participants did not experience VF in their first 36 months of HIV treatment. Our observed rate of VF (8.4\%) over three years is in line with the 90-90-90 goals set forth by the Joint United Nations Programme for HIVIAIDS (UNAIDS) [15]. Nearly $20 \%$ of those who experienced VF had no detectable resistance mutations, suggesting nonadherence with minimal drug exposure. Of the remaining $80 \%$ with antiretroviral drug resistance, the majority of the resistance mutations could be detected by the time of VF, which increased further with prolonged time on a failing first-line ART regimen.

The rates of VF observed in our pragmatic study were encouraging and similar to other reports from the region. A recent surveillance study from Rwanda found $12 \%$ of ART initiators experienced VF at 12 months [16]. In ANRS 12249 trial, based in South Africa, high rates of 12-month viral suppression (97\%) were achieved using a combination of TDF+FTC+EFV as first-line therapy [17]. That our study had a slightly higher estimate for VF was not surprising, given the "real world" clinical settings we sought to replicate and longer follow up. Early during study enrollment, in accordance with Zambian treatment 
This pdf is for personal use only. To obtain commercial reprints, please contact the editorial office on +442073980700

Publication: Antiviral Therapy; Type: Original article DOI: 10.3851/IMP3299

guidelines at the time, we relied heavily on older ART regimens that incorporated NVP and non-TDF NRTIs, agents that may be less durable than currently recommended first-line regimens, especially at higher viral loads [18].

Using Sanger sequencing, we observed a high prevalence of pretreatment drug resistance among individuals with eventual VF, particularly for NNRTIs (23\%). We did not perform baseline genotypes on participants who did not experience VF; as a result, we were unable to directly compare pretreatment drug resistance on VF. However, this prevalence is higher than what has been observed in other regional studies including the most recent regional estimates (8-15\%) in the 2017 World Health Organization Drug Resistance report [4]. Several factors may have contributed to the high levels of pretreatment NNRTI drug resistance in our sample. First, as we only have pretreatment data on those with virologic failure, we may have selected the group with higher levels of pre-treatment drug resistance. Interestingly, the rate of VF was not significantly different between those with NRTI or NNRTI baseline resistance and those without. The ANRS 12249 trial in South Africa had similar findings: no association between pre-treatment drug resistance and virologic suppression was detected over 16 months [17]. Other explanations include an older national program, which has been associated with higher levels of transmitted resistance [19]; we did not see an association between time of enrollment and baseline resistance. The prevalent use of peripartum NVP for the prevention of mother-to-child HIV transmission part of the standard of care for many years in Zambia - also likely contributed to the higher levels of pretreatment NNRTI resistance [20].

By the time VF was detected, many of the key damaging mutations had already accumulated. This finding suggests that WHO recommended six-month interval for viral load monitoring may not be optimal. There is often further delay for the turnaround time for results to reach the referring facility between 3 and 31 days in one recent study of seven sub-Saharan African countries [21]. These data do not account for further delays for the result to be acted upon clinically. Further implementation research should focus on the optimal use of viral load monitoring.

Our results also highlight the need for interventions that prevent VF and thus resistance. Similar to our findings, a recently published report from Lesotho found that $85 \%$ of adults failed first-line therapy with detectable resistance mutations [22]. Of those adults with a detectable viral load after 6 months of ART, 64\% remained unsuppressed after an expanded adherence counseling intervention. HIV treatment facilities and the research community should focus on tools for early detection of poor adherence and implementation of effective, scalable interventions to improve adherence with a renewed focus on preserving first-line therapy. The Bill and Melinda Gates Foundation recently published a summary of the most promising, scalable interventions including peer counseling, adherence clubs, and short message service (SMS) possibly paired to electronic dose monitors [23]. The hope of expanded access to viral load testing, including point of care viral load testing, to improve early detection of non-suppressed patients will require effective adherence interventions to help those patients achieve viral suppression after early 
This pdf is for personal use only. To obtain commercial reprints, please contact the editorial office on +442073980700

Publication: Antiviral Therapy; Type: Original article DOI: 10.3851/IMP3299

detection. The need to adapt such adherence packages to local settings must be weighed against other competing priorities within the UNAIDS 90-90-90 goals.

Our study offered a unique opportunity to observe the accumulation of HIV resistance mutations among patients on failing first-line therapy. Over considerable intervals between VF and the last available genotype (median 202 days; IQR 139-461), we observed modest increases in antiretroviral resistance. Predicted TDF resistance increased from $42 \%$ to $58 \%$ in those failing TDF-based regimens, similar to the TenoRes study and a recently published meta-analysis of tenofovir-based first-line failures in subSaharan Africa [7,24]. TAMs increased more than NNRTI resistance mutations with prolonged failing on first line regimen. For those on $\mathrm{d} 4 \mathrm{~T}$-based regimens, predicted tenofovir resistance did not change. In a small cohort of 26 patients with subtype $C$ virus followed 6-12 months after VF on d4T or AZT regimens, K65R mutations doubled from $12 \%$ to $23 \%$ [25]; Predicted TDF resistance doubled from roughly $20 \%$ to $40 \%$, which is higher than another recently reported small study from Mozambique [26]. A slightly larger study of 63 adults including 28 with sub-type $C$ and 18 on tenofovir based ART showed that resistance to tenofovir and zidovudine increased from $10 \%$ to $30 \%$ with continued VF, however resistance on tenofovirbased ART or the emergence of TAMs while on tenofovir was not reported [6].

The resistance patterns observed in our last available genotypes could threaten Zambia's recently reported success of $90 \%$ viral suppression among those on ART [4]. The NNRTI resistance of $87 \%$ to efavirenz is particularly concerning for the potential for transmitted drug resistance to first-line therapy; as such, increasing rates of pretreatment drug resistance has led international agencies to consider integrase-based first-line therapy in certain countries [4]. Further it may compromise the effectiveness of future injectable NNRTIs. Importantly, acquired NRTI mutations also affect second-line ART options [27]. Fifteen percent of those taking TDF-based regimens developed 2 or more TAMs by VF. This finding was unexpected since tenofovir has not traditionally been associated with TAM emergence [24]; however, it was similarly observed in secondary analysis of the TenoRes collaboration. In that study it was attributed to substitutions from prior ART, however in our study ART naive participants initiated first line ART; this finding should be further evaluated in future studies of sub-type $C$. There is ongoing debate over the roles of resistance and adherence in second-line failure since no second-line ART randomized trials have achieved 90\% viral suppression [27-30]. Interestingly, two clinical trials of second-line regimens enrolled participants of whom over $95 \%$ had baseline NNRTI resistance $[29,30]$, compared to the $80 \%$ NNRTI resistance at VF observed in our cohort, which suggests that $20 \%$ were non-adherent enough not to get resistance versus less than $5 \%$ in second-line trials. This discrepancy suggests that enrollment into these second-line trials may select for more adherent participants and may not be representative of the general population failing first line ART regimens. The benefits of treatment as prevention are less likely to accrue if second-line therapy does not achieve full viral suppression $[27,28]$.

Strengths of this study include its large sample size, its prospective design, and lengthy longitudinal follow-up. Despite the older treatment regimens, including ones incorporating d4T and NVP, 
This pdf is for personal use only. To obtain commercial reprints, please contact the editorial office on +442073980700

Publication: Antiviral Therapy; Type: Original article DOI: 10.3851/IMP3299

our results are still relevant to understanding the evolution of resistance mutations and potential circulating resistance patterns in Zambia. Our findings are also relevant to second- and third-line regimens in settings where the majority of viruses are subtype $\mathrm{C}$. We acknowledge limitations as well. For example, HIV resistance tests were performed retrospectively, as dictated by the protocol, which contributed to missing genotype results, thus we chose to analyze new mutations at key time points rather than individual accumulation rates. No genotypes were run on those that did not have VF limiting our analysis of pretreatment drug resistance. Samples could not be repeated if volumes were found to be inadequate or if specimens were lost. As with all genotype assays, we were limited by the lower limit of detection for viral concentration; we are unable to comment on those individuals who were not virologically suppressed but had less than 1,000 viral copies per $\mathrm{mL}$. We relied on consensus sequencing, which can only detect resistance mutations comprising $>25 \%$ of the circulating viral population [31]. Because of this effect, our results are likely a conservative estimate of the true resistance that may be present. Newer techniques can double the detection of resistance in minor variants [17]. Recent data also suggests that genotype might not predict phenotype as well for second-generation NNRTIs in sub-type C HIV-1 infection [32].

In summary, in this study of virologic failure on first-line ART in Zambia, we found significant resistance to NRTIs and NNRTIs at the time of virologic failure, which increased with prolonged failing on first-line regimens. These data highlight the urgent need for effective adherence strategies, earlier detection of VF including distinguishing poor adherence from resistance, and access to newer drug classes. Each of these challenges must be addressed if the ambitious 90-90-90 goals set forth by international agencies and local governments are to be met.

\section{Acknowledgements}

We thank Suwilanji Silwamba for technical assistance with HIV sequencing. This study was supported by the President's Emergency Plan for AIDS Relief (PEPFAR) through a multicountry grant to the Elizabeth Glaser Pediatric AIDS Foundation from the U.S. Centers for Disease Control and Prevention (cooperative agreement U62/CCU12354) and an administrative supplement to the U.S. National Institutes of Health (U01 Al069452). Additional support for investigators, trainees, and research administration was provided through the National Institutes of Health (T32 Al007001, K24 Al120796, P30 Al050410, P30 Al027767). The funders had no role in the design, analysis or writing of this article.

This study was presented as poster 537 at Conference on Retroviruses and Opportunistic Infections in March 2018 in Boston, MA.

\section{Declaration of Interests:}

We have no conflicts of interest to report.

\section{References}

1. Global action plan on HIV drug resistance 2017-2021. Geneva: World Health Organization; 2017.

2. Consolidated guidelines on the use of antiretroviral drugs for treating and preventing HIV infection: recommendations for a public health approach - 2nd ed. Geneva: World Health Organization; 2016. 
This pdf is for personal use only. To obtain commercial reprints, please contact the editorial office on +442073980700

Publication: Antiviral Therapy; Type: Original article DOI: 10.3851/IMP3299

3. Phillips AN, Stover J, Cambiano V, et al. Impact of HIV Drug Resistance on HIVIAIDS-Associated Mortality, New Infections, and Antiretroviral Therapy Program Costs in Sub-Saharan. Afr J Infect Dis 2017; 215:1362-1365.

4. $\quad$ HIV drug resistance report 2017. Geneva: World Health Organization; 2017.

5. Hamers RL, Sigaloff KC, Wensing AM, et al. Patterns of HIV-1 drug resistance after first-line antiretroviral therapy (ART) failure in 6 sub-Saharan African countries: implications for second-line ART strategies. Nephrol Dial Transplant 2012; 54:1660-1669.

6. Boender TS, Kityo CM, Boerma RS, et al. Accumulation of HIV-1 drug resistance after continued virological failure on first-line ART in adults and children in sub-Saharan Africa. $J$ Antimicrob Chemother 2016; 71:2918-2927.

7. TenoRes Study Group. Global epidemiology of drug resistance after failure of WHO recommended first-line regimens for adult HIV-1 infection: a multicentre retrospective cohort study. Lancet Infect Dis 2016; 16:565-575.

8. Reynolds SJ, Sendagire $\mathrm{H}$, Newell $\mathrm{K}$, et al. Virologic versus immunologic monitoring and the rate of accumulated genotypic resistance to first-line antiretroviral drugs in Uganda. BMC Infect Dis 2012; 12:381.

9. Kityo C, Thompson J, Nankya I, Hoppe A, Ndashimye E, Warambwa C, et al. HIV Drug Resistance Mutations in Non-B Subtypes After Prolonged Virological Failure on NNRTI-Based First-Line Regimens in Sub-Saharan Africa. Journal of acquired immune deficiency syndromes (1999). 2017;75(2):e45-e54.

10. Koethe JR, Westfall AO, Luhanga DK, et al. A cluster randomized trial of routine HIV-1 viral load monitoring in Zambia: study design, implementation, and baseline cohort characteristics. PLoS One 2010; 5:e9680.

11. Saag MS, Westfall A, Luhanga DK, et al. (Editors). A cluster-randomized trial of routine vs. discretionary viral load monitoring among adults starting ART: Zambia [Abstract 87]. 19th Conference on Retroviruses and Opportunistic Infections 2012; Seattle, WA.

12. Goldman JD, Cantrell RA, Mulenga LB, et al. Simple adherence assessments to predict virologic failure among HIV-infected adults with discordant immunologic and clinical responses to antiretroviral therapy. AIDS Res Hum Retroviruses 2008; 24:1031-1035.

13. Seu L, Mulenga LB, Siwingwa M, et al. Characterization of HIV drug resistance mutations among patients failing first-line antiretroviral therapy from a tertiary referral center in Lusaka, Zambia. $J$ Med Virol 2015; 87:1149-1157.

14. Tang MW, Rhee SY, Bertagnolio S, et al. Nucleoside reverse transcriptase inhibitor resistance mutations associated with first-line stavudine-containing antiretroviral therapy: programmatic implications for countries phasing out stavudine. J Infect Dis 2013; 207 Suppl 2:S70-S77.

15. 90-90-90 An ambitious treatment target to help end the AIDS epidemic. Geneva: Joint United Nations Programme on HIVIAIDS (UNAIDS); 2014.

16. Ndahimana J, Riedel DJ, Mwumvaneza M, et al. Drug resistance mutations after the first 12 months on antiretroviral therapy and determinants of virological failure in Rwanda. Tropical medicine \& international health. $T M \& I H$. 2016; 21:928-935.

17. Derache A, Iwuji C, Danaviah S, et al. (Editors). Prevalence and impact of pretreatment drug resistance in the ANRS 12249 TASP Trial [Abstract 43]. 2017 Conference on Retroviruses and Opportunistic Infections; 2017; Seattle, WA.

18. Willig $\mathrm{JH}$, Abroms $\mathrm{S}$, Westfall $\mathrm{AO}$, et al. Increased regimen durability in the era of once-daily fixed-dose combination antiretroviral therapy. AIDS 2008; 22:1951-1960.

19. Hamers RL, Wallis CL, Kityo C, et al. HIV-1 drug resistance in antiretroviral-naive individuals in sub-Saharan Africa after rollout of antiretroviral therapy: a multicentre observational study. Lancet Infect Dis 2011; 11:750-759. 
This pdf is for personal use only. To obtain commercial reprints, please contact the editorial office on +442073980700

Publication: Antiviral Therapy; Type: Original article DOI: 10.3851/IMP3299

20. Arrive E, Newell ML, Ekouevi DK, et al. Prevalence of resistance to nevirapine in mothers and children after single-dose exposure to prevent vertical transmission of HIV-1: a meta-analysis. Int J Epidemiol 2007; 36:1009-1021.

21. Lecher S, Ellenberger D, Kim AA, et al. Scale-up of HIV Viral Load Monitoring--Seven SubSaharan African Countries. MMWR Morb Mortal Wkly Rep 2015; 64:1287-1290.

22. Labhardt ND, Bader J, Lejone TI, et al. Should viral load thresholds be lowered?: Revisiting the WHO definition for virologic failure in patients on antiretroviral therapy in resource-limited settings. Medicine (Baltimore) 2016; 95:e3985.

23. Haberer JE, Sabin L, Amico KR, et al. Improving antiretroviral therapy adherence in resourcelimited settings at scale: a discussion of interventions and recommendations. J Int AIDS Soc 2017; 20:21371.

24. Gregson J, Kaleebu P, Marconi VC, et al. Occult HIV-1 drug resistance to thymidine analogues following failure of first-line tenofovir combined with a cytosine analogue and nevirapine or efavirenz in sub Saharan Africa: a retrospective multi-centre cohort study. Lancet Infect Dis 2017; 17:296-304.

25. Barth RE, Aitken SC, Tempelman $\mathrm{H}$, et al. Accumulation of drug resistance and loss of therapeutic options precede commonly used criteria for treatment failure in HIV-1 subtype-C-infected patients. Antivir Ther 2012; 17:377-386.

26. De Luca A, Sidumo ZJ, Zanelli G, et al. Accumulation of HIV-1 drug resistance in patients on a standard thymidine analogue-based first line antiretroviral therapy after virological failure: implications for the activity of next-line regimens from a longitudinal study in Mozambique. BMC Infect Dis 2017; 17:605.

27. Stockdale AJ, Saunders MJ, Boyd MA, et al. Effectiveness of Protease Inhibitor/Nucleos(t)ide Reverse Transcriptase Inhibitor-Based Second-line Antiretroviral Therapy for the Treatment of Human Immunodeficiency Virus Type 1 Infection in Sub-Saharan Africa: A Systematic Review and Meta-analysis. Nephrol Dial Transplant 2018; 66:1846-1857.

28. Paton NI, Kityo C, Hoppe A, et al. Assessment of second-line antiretroviral regimens for HIV therapy in Africa. N Engl J Med 2014; 371:234-247.

29. Group S-LS, Boyd MA, Kumarasamy N, Moore CL, Nwizu C, Losso MH, et al. Ritonavir-boosted lopinavir plus nucleoside or nucleotide reverse transcriptase inhibitors versus ritonavir-boosted lopinavir plus raltegravir for treatment of HIV-1 infection in adults with virological failure of a standard first-line ART regimen (SECOND-LINE): a randomised, open-label, non-inferiority study. Lancet 2013; 381:2091-2099.

30. La Rosa AM, Harrison LJ, Taiwo B, et al. Raltegravir in second-line antiretroviral therapy in resource-limited settings (SELECT): a randomised, phase 3, non-inferiority study. Lancet HIV 2016; 3:e247-e258.

31. Schuurman R, Demeter L, Reichelderfer P, Tijnagel J, de Groot T, Boucher C. Worldwide evaluation of DNA sequencing approaches for identification of drug resistance mutations in the human immunodeficiency virus type 1 reverse transcriptase. J Clin Microbiol 1999; 37:2291-2296.

32. Derache A, Wallis CL, Vardhanabhuti S, Bartlett J, Kumarasamy N, Katzenstein D. Phenotype, Genotype, and Drug Resistance in Subtype C HIV-1 Infection. J Infect Dis 2016; 213:250-256. 
This pdf is for personal use only. To obtain commercial reprints, please contact the editorial office on +442073980700

Publication: Antiviral Therapy; Type: Original article DOI: 10.3851/IMP3299

Figures

Figure 1. Flowchart of the 165 Participants with Virologic Failure and Their 464 Genotype Tests

Figure 2. Time to Virologic Failure Following Anti-retroviral Therapy Initiation, Stratified by Any Major NRTI or NNRTI Mutations on Baseline Genotype

Figure 3. Predicted Drug Resistance at Baseline, Virologic Failure, and Last Available Genotype

Drug resistance predicted by Stanford HIV Drug Resistance Database (v8.2). Baseline (BL) was prior to initiation of first-line ART. Virologic failure (VF) was run on the first viral load sample except in 5 cases when it was run on the confirmatory viral load within 90 days. Last available genotype (LG).

Median time (IQR) from BL to VF: 356 days (IQR: 175-553)

Median time (IQR) from VF to LG: 202 days (IQR: 139-461

Supplement Figure. Predicted Drug Resistance at Baseline, Virologic Failure, and Last Available Genotype by Study Arm of the Parent, Cluster Randomized Trial

Median time (IQR) from BL to VF: 356 days (IQR: 175-553) Median time (IQR) from VF to LG: 202 days (IQR: 139-

461)

Control arm with clinical monitoring, Intervention arm with routine viral load monitoring

Supplement Table 2a. Cumulative Reverse Transcriptase Mutations on Failing First Line Antiretroviral Therapy, by Monitoring Arm of Parent, Cluster Randomized Trial

Supplemental Table 2b: Cumulative Reverse Transcriptase Mutations on Failing First Line Antiretroviral Therapy with Prolonged Virologic Failure 
This pdf is for personal use only. To obtain commercial reprints, please contact the editorial office on +442073980700

Publication: Antiviral Therapy; Type: Original article DOI: $10.3851 /$ IMP3299

Table 1: Baseline Characteristics

\begin{tabular}{|c|c|c|c|c|}
\hline \multirow[t]{2}{*}{ Characteristics } & \multicolumn{2}{|c|}{$\begin{array}{l}\text { All participants in the } \\
\text { parent cluster- } \\
\text { randomized trial } \\
(n=1973)\end{array}$} & \multicolumn{2}{|c|}{$\begin{array}{l}\text { Participants experiencing } \\
\text { virologic failure } \\
(n=165)\end{array}$} \\
\hline & $\mathbf{n}$ & $\begin{array}{l}\text { \% or median } \\
\text { (IQR) }\end{array}$ & $\mathbf{n}$ & $\%$ or median (IQR) \\
\hline Age & 1973 & $34.3(29.4-39.9)$ & 165 & $32.1(27.7-38.4)$ \\
\hline Female & 1182 & 60 & 100 & 61 \\
\hline $\begin{array}{l}\text { Prior drug exposure from PMTCT } \\
\text { (women only) }\end{array}$ & 193 & 19 & 28 & 33 \\
\hline Body mass index & 1941 & $20(18-22)$ & 164 & $20(19-22)$ \\
\hline Cockcroft-Gault $\mathrm{Cr} \mathrm{Cl}<60 \mathrm{~mL} / \mathrm{m}^{2}$ & 64 & 3.5 & 3 & 2 \\
\hline Hemoglobin concentration $(\mathrm{mg} / \mathrm{dL})$ & 1904 & $10.9(9.6-12.3)$ & 163 & $11.1(9.9-12.0)$ \\
\hline Active tuberculosis & 373 & 19 & 36 & 22 \\
\hline WHO Stage III or IV & 1283 & 68 & 98 & 63 \\
\hline CD4 count (cells $/ \mathrm{mm}^{3}$ ) & 1907 & $139(79-192)$ & 160 & $126(57-182)$ \\
\hline$\geq 200$ & 418 & 22 & 28 & 18 \\
\hline $50-199$ & 1233 & 65 & 99 & 62 \\
\hline$<50$ & 256 & 21 & 33 & 21 \\
\hline Viral Load $>100,000$ copies $/ \mathrm{mL}$ & 1065 & 54 & 94 & 57 \\
\hline Sub-type C & $\#$ & $\#$ & 154 & 93 \\
\hline NRTI component & 1972 & & 164 & \\
\hline ZDV-based regimen & 849 & 43 & 84 & 51 \\
\hline d4T-based regimen & 658 & 33 & 53 & 32 \\
\hline TDF-based regimen & 465 & 24 & 27 & 16 \\
\hline NNRTI component & 1973 & & 165 & \\
\hline EFV-based regimen & 478 & 24 & 26 & 16 \\
\hline NVP-based regimen & 1495 & 76 & 139 & 84 \\
\hline Assigned to intervention arm & 989 & 50 & 79 & 48 \\
\hline
\end{tabular}

\# Only participants with virologic failure were sub-typed

Total Missing Data: PMTCT 161, BMI 32, Cr Cl 148, Hgb 69, WHO Stage 86, CD4 66, VL 5, NRTI 1

VF Missing Data: BMI 1, Cr Cl 15, Hgb 2, WHO Stage 9, CD4 5, NRTI 1

Prevention mother to child transmission (PMTCT), body mass index (BMI), creatinine clearance $(\mathrm{Cr} \mathrm{Cl})$, nucleoside reverse transcriptase inhibitor (NRTI), non-nucleoside reverse transcriptase inhibitor (NNRTI), zidovudine (ZDV), stavudine (d4T), tenofovir disoproxil fumarate (TDF), efavirenz (EFV), nevirapine (NVP) 
This pdf is for personal use only. To obtain commercial reprints, please contact the editorial office on +442073980700

Publication: Antiviral Therapy; Type: Original article DOI: 10.3851/IMP3299

Table 2: Cumulative Reverse Transcriptase Mutations on Failing First Line Antiretroviral Therapy

\begin{tabular}{|lccc|}
\hline Mutations & $\begin{array}{c}\text { Treatment } \\
\text { Initiation (BL) } \\
\mathrm{n}=132\end{array}$ & $\begin{array}{c}\text { Virologic Failure } \\
(\text { VF) } \\
\mathrm{n}=116\end{array}$ & $\begin{array}{c}\text { Last Available } \\
\text { Genotype (LG) } \\
\mathrm{n}=151\end{array}$ \\
\hline & $\mathrm{n}(\%)$ & $\mathrm{n}(\%)$ & $\mathrm{n}(\%)$ \\
\hline NRTI & $13(10)$ & $75(65)$ & $115(76)$ \\
\hline Any NRTI & $3(2)$ & $68(59)$ & $107(71)$ \\
\hline M184 & $2(2)$ & $13(11)$ & $20(13)$ \\
\hline K65R & $1(1)$ & $3(3)$ & $18(12)$ \\
\hline TAMs $>1$ & $30(23)$ & $94(81)$ & $131(87)$ \\
\hline NNRTI & $13(10)$ & $43(37)$ & $65(43)$ \\
\hline Any NNRTI & $9(7)$ & $37(32)$ & $56(37)$ \\
\hline K103N & $1(1)$ & $10(8.6)$ & $14(9)$ \\
\hline Y181CIV & 0 & $23(20)$ & $39(26)$ \\
\hline Y188LCH & $12(9)$ & $67(58)$ & $96(64)$ \\
\hline G190 ASEQ & $7(5)$ & $74(64)$ & $112(74)$ \\
\hline $\begin{array}{l}\text { Pan-NNRTI: L100I, } \\
\text { K101EP, Y181, } \\
\text { Y188L, G190, M230L }\end{array}$ & $36(27)$ & $95(82)$ & $134(89)$ \\
\hline Any NRTI + NNRTI & & & \\
\hline Any NRTI or NNRTI & & & \\
\hline
\end{tabular}

Nucleoside reverse transcriptase inhibitor (NRTI), non-nucleoside reverse transcriptase inhibitor (NNRTI), thymidine analog mutations (TAMs) 


\section{Missing Genotype Tests}

No Baseline Genotype: 33
Below threshold: 13
Technical failure: 16
No storage: 4

No VF Genotype: 49
Below threshold: 25
Technical failure: 20
No storage: 4

No 6mo Genotype: 31
Below threshold: 17
Technical failure: 10
No storage: 4

No 12mo Genotype: 35 Below threshold: 18 Technical failure: 7 No VL: 10

No 18mo Genotype: 24
Below threshold: 8
Technical failure: 3
No VL: 13

No 24mo Genotype: 16
Below threshold: 6
Technical failure: 2
No VL: 8

\section{Randomized $n=1973$}

\section{Censored Without Genotype}

This pdf is for personal use only. To obtain commercial reprints, please contact the editorial office on +44 2073980700
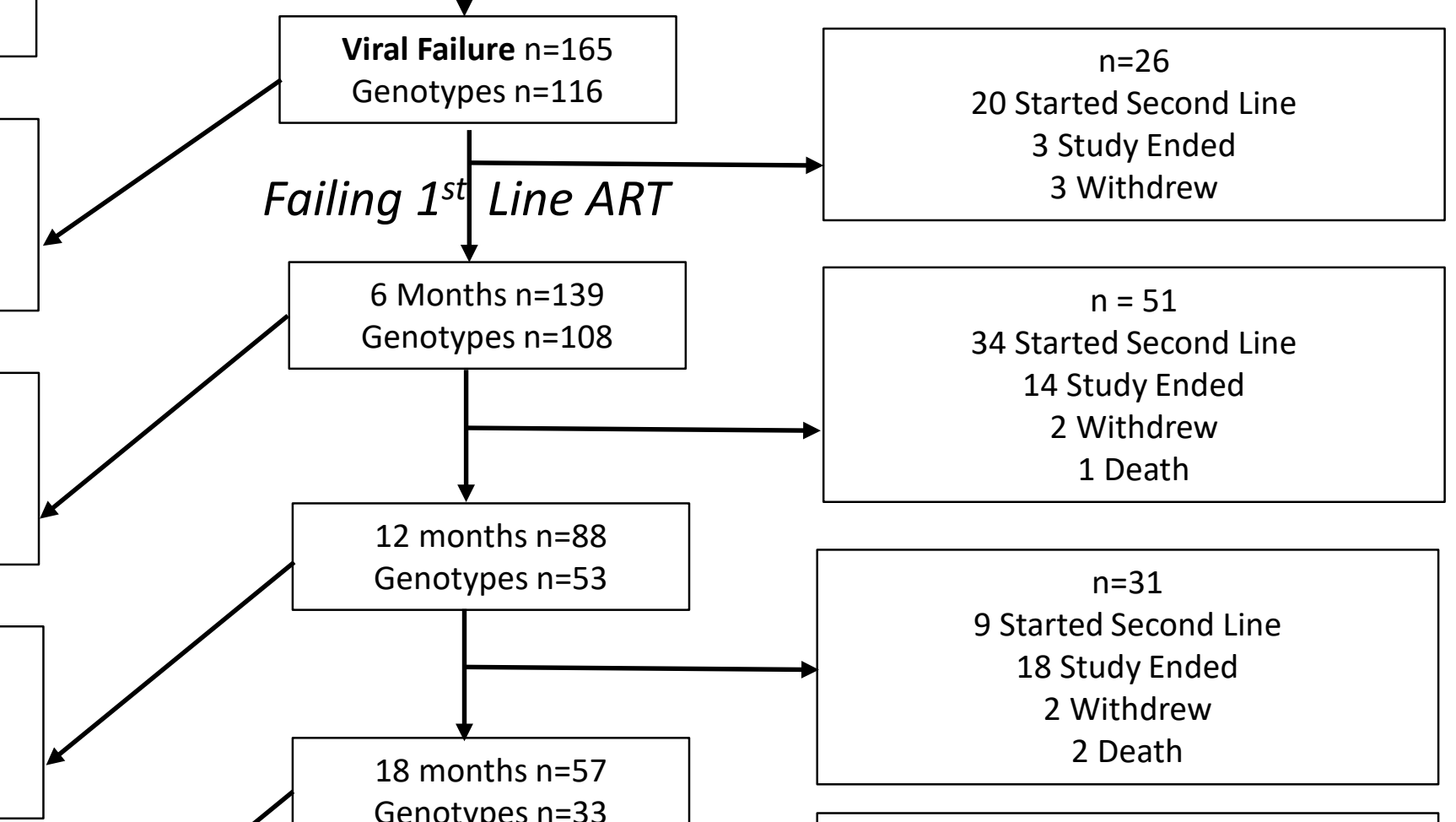

\section{$\mathrm{n}=28$}

13 Started Second Line

12 Study Ended

3 Withdrew

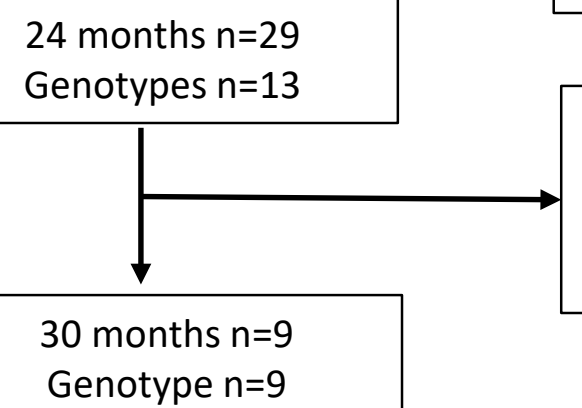

3 Started Second Line 16 Study Ended

1 Withdrew 
This pdf is for personal use only. To obtain commercial reprints,

please contact the editorial office on +442073980700

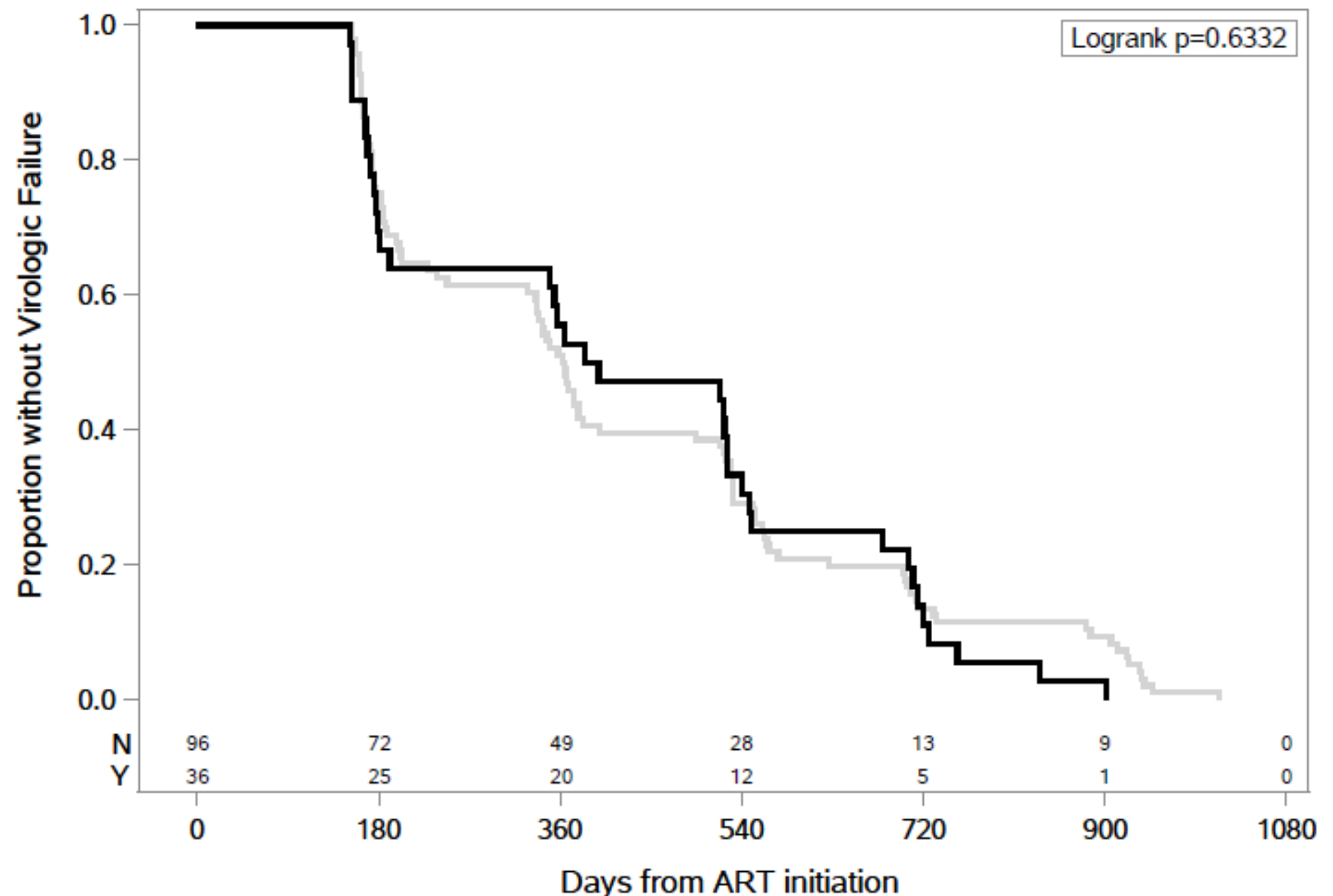

NRTI or NNRTI mutation at $\mathrm{BL}=\mathrm{N}=\mathrm{Y}$ 


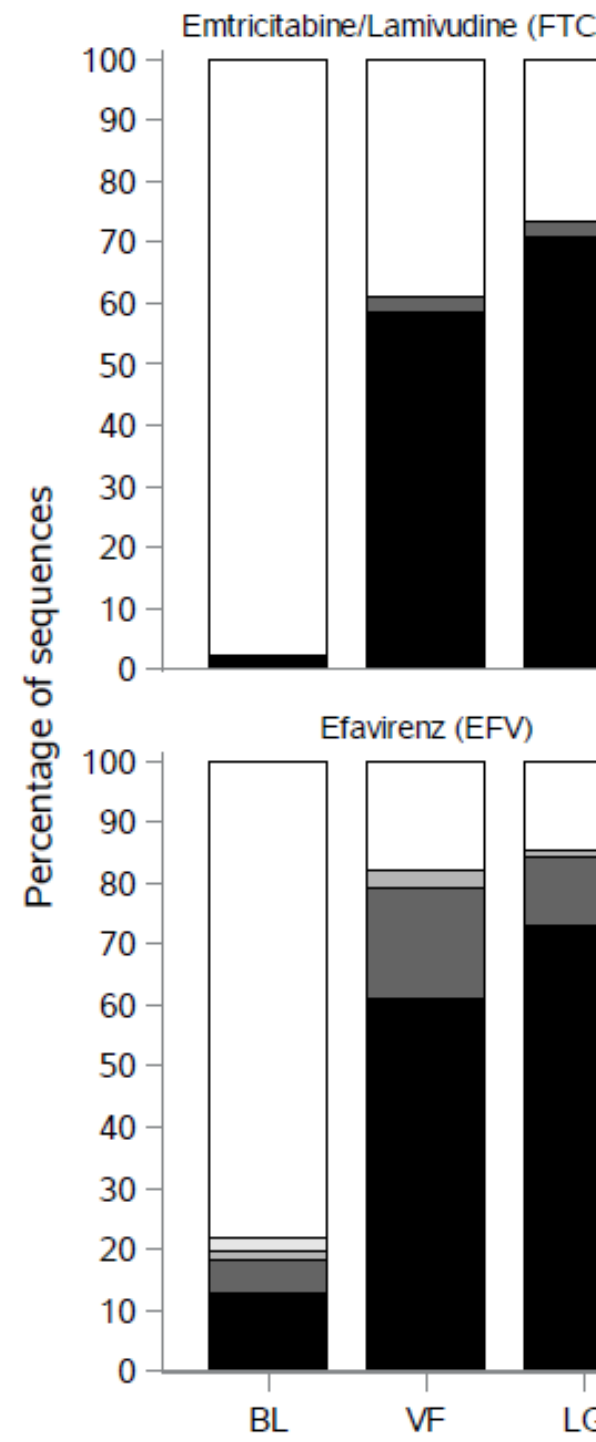

Zidovudine (ZDV)

Tenofovir (TDF)

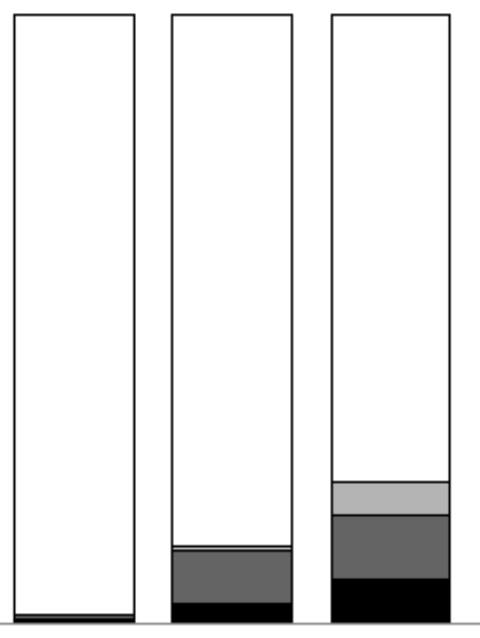

Etravirine (ETR)

Nevirapine (NVP)

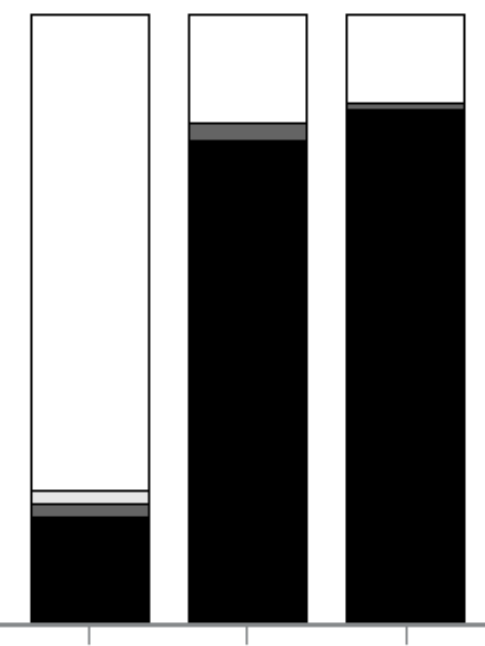

$\mathrm{BL}$

VF

LG

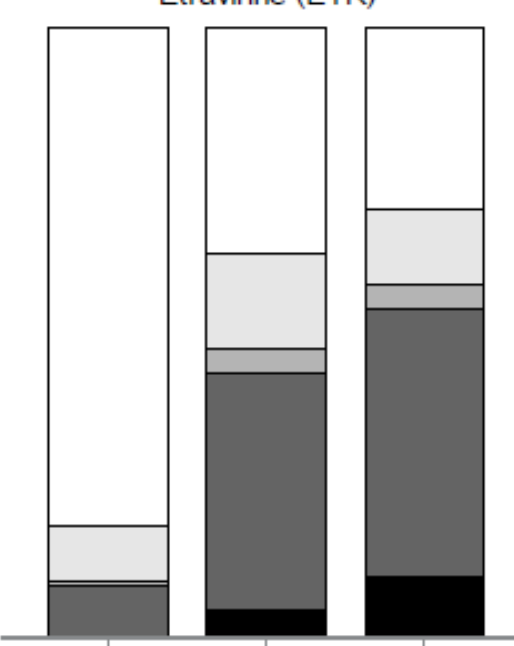

VF
Abacavir (ABC)

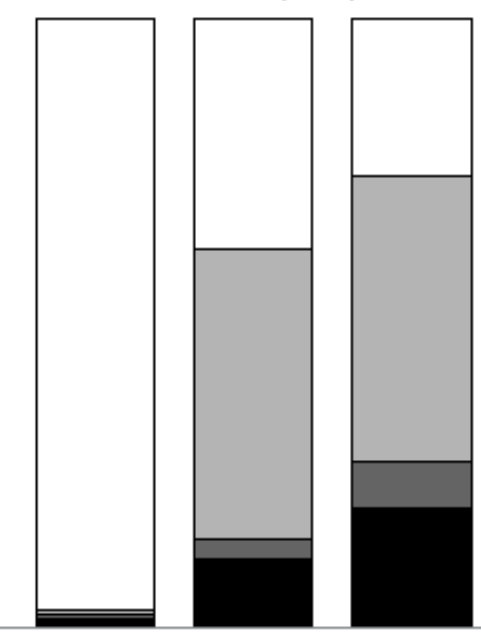

Rilpivirine (RPV)

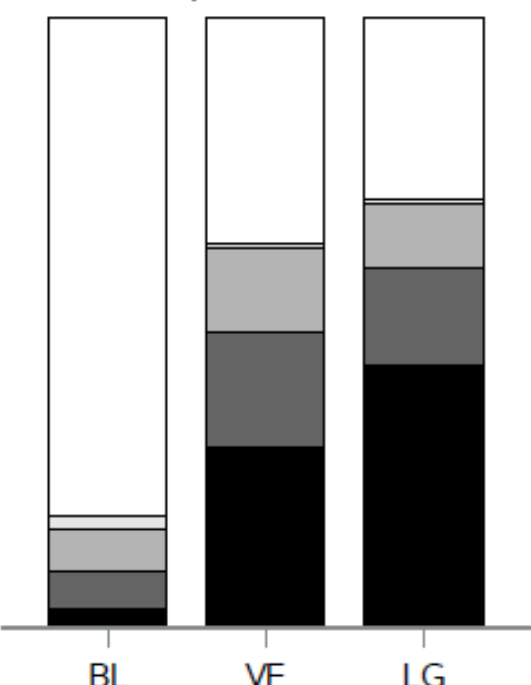

$\square$ Susceptible $\square$ Potential low-level resistance $\square$ Low-level resistance $\square$ Intermediate resistance $\quad \square$ High-level resistance

This pdf is for personal use only. To obtain commercial reprints, please contact the editorial office on +442073980700 
Emtricitabine/Lamivudine (FTC/3TC)

Tenofovir (TDF)

Abacavir (ABC)

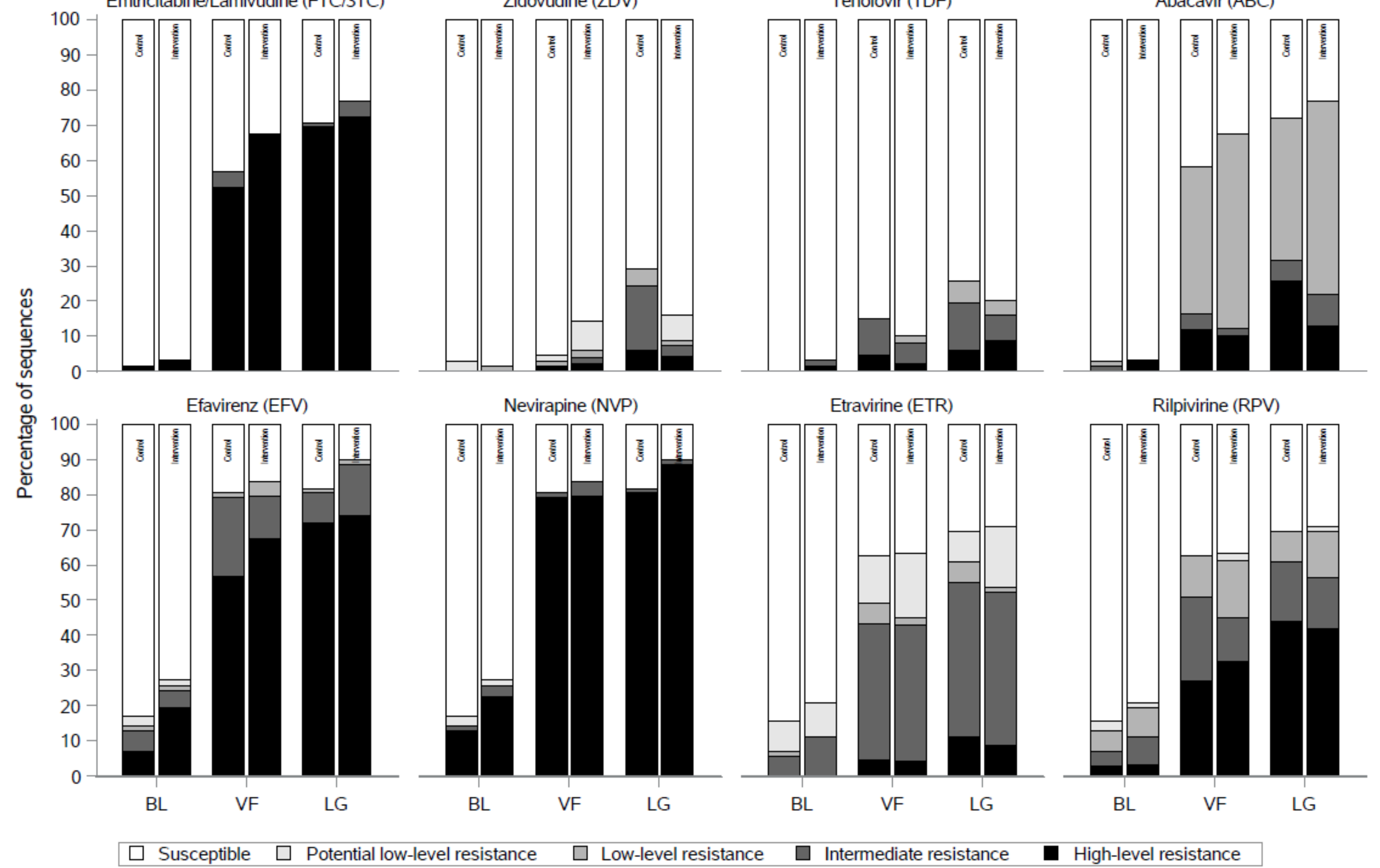

Median time (IQR) from BL to VF: 356 days (IQR: 175-553) Median time (IQR) from VF to LG: 202 days (IQR: 139-461) Control arm with clinical monitoring, Intervention arm with routine viral load monitoring 
This pdf is for personal use only. To obtain commercial reprints, please contact the editorial office on +442073980700

Supplement Table 2a: Cumulative Reverse Transcriptase Mutations on Failing First Line Antiretroviral Therapy, by Monitoring Arm of Parent, Cluster Randomized Trial

\begin{tabular}{|c|c|c|c|c|c|c|}
\hline \multirow[t]{2}{*}{ Mutations } & \multicolumn{2}{|c|}{$\begin{array}{l}\text { Treatment Initiation } \\
\text { (BL) } \\
n=132\end{array}$} & \multicolumn{2}{|c|}{$\begin{array}{l}\text { Virologic Failure } \\
\qquad \begin{array}{c}\text { (VF) } \\
n=116\end{array}\end{array}$} & \multicolumn{2}{|c|}{$\begin{array}{l}\text { Last Available Genotype } \\
\text { (LG) } \\
\mathrm{n}=151\end{array}$} \\
\hline & $\begin{array}{c}\text { Control } \\
n=70\end{array}$ & $\begin{array}{l}\text { Interv. } \\
\mathrm{n}=62\end{array}$ & $\begin{array}{c}\text { Control } \\
\mathrm{n}=67\end{array}$ & $\begin{array}{l}\text { Interv. } \\
n=49\end{array}$ & $\begin{array}{c}\text { Control } \\
\mathrm{n}=82\end{array}$ & $\begin{array}{c}\text { Interv. } \\
\mathrm{n}=69\end{array}$ \\
\hline NRTI & n (\%) & n (\%) & n (\%) & n (\%) & n (\%) & n (\%) \\
\hline Any NRTI & $9(13)$ & $4(7)$ & $40(60)$ & $35(71)$ & $59(72)$ & $56(81)$ \\
\hline M184 & $1(1)$ & $2(3)$ & $35(52)$ & $33(67)$ & $57(70)$ & $50(72)$ \\
\hline K65R & $0(0)$ & $2(3)$ & $9(13)$ & $4(8)$ & $10(12)$ & $10(14)$ \\
\hline TAMs $>1$ & $1(1)$ & $0(0)$ & $1(1)$ & $2(4)$ & $13(16)$ & $5(7)$ \\
\hline \multicolumn{7}{|l|}{ NNRTI } \\
\hline Any NNRTI & $13(19)$ & $17(27)$ & $55(82)$ & $39(80)$ & $69(84)$ & $62(90)$ \\
\hline $\mathrm{K} 103 \mathrm{~N}$ & $4(6)$ & $9(15)$ & $21(31)$ & $22(45)$ & $31(38)$ & $34(49)$ \\
\hline Y181CIV & $3(4)$ & $6(10)$ & $22(33)$ & $15(31)$ & 30 (37) & $26(38)$ \\
\hline Y188LCH & $0(0)$ & $1(2)$ & $4(6)$ & $6(12)$ & $5(6)$ & $9(13)$ \\
\hline G190 ASEQ & $0(0)$ & $0(0)$ & $15(22)$ & $8(16)$ & $23(28)$ & $16(23)$ \\
\hline $\begin{array}{l}\text { Pan-NNRTI: L100I, K101EP, } \\
\text { Y181, Y188L, G190, M230L }\end{array}$ & $5(7)$ & 7 (11) & $41(61)$ & $26(53)$ & $52(63)$ & $44(64)$ \\
\hline Any NRTI + NNRTI & $4(6)$ & $3(5)$ & $40(60)$ & $34(70)$ & $58(71)$ & $54(78)$ \\
\hline Any NRTI or NNRTI & $18(26)$ & 18 (29) & $55(82)$ & $40(82)$ & 70 (85) & $64(93)$ \\
\hline
\end{tabular}

Control arm with clinical monitoring, Intervention arm with routine viral load monitoring

Nucleoside reverse transcriptase inhibitor (NRTI), non-nucleoside reverse transcriptase inhibitor (NNRTI), thymidine analog mutations (TAMs) 
This pdf is for personal use only. To obtain commercial reprints, please contact the editorial office on +442073980700

Supplemental Table 2b: Cumulative Reverse Transcriptase Mutations on Failing First Line Antiretroviral Therapy with Prolonged Virologic Failure

\begin{tabular}{|c|c|c|c|c|c|}
\hline Mutations & $\begin{array}{c}\text { Treatment } \\
\text { Initiation }(\mathrm{BL}) \\
\mathrm{n}=132\end{array}$ & $\begin{array}{l}\text { Virologic Failure } \\
\text { (VF) } \\
n=116\end{array}$ & $\begin{array}{l}\text { Last Available } \\
\text { Genotype (LG) } \\
\text { n=151 }\end{array}$ & $\begin{array}{c}\mathrm{VF} \geq 9 \text { months } \\
\mathrm{n}=63\end{array}$ & $\begin{array}{c}\mathrm{VF} \geq 15 \text { months } \\
\mathrm{n}=38\end{array}$ \\
\hline \multicolumn{6}{|l|}{ NRTI } \\
\hline Any NRTI & $13(10)$ & $75(65)$ & $115(76)$ & $54(86)$ & $33(87)$ \\
\hline K65R & $2(2)$ & $13(11)$ & $20(13)$ & $9(14)$ & $7(18)$ \\
\hline TAMs $>1$ & $1(1)$ & $3(3)$ & $18(12)$ & $11(17)$ & $9(24)$ \\
\hline \multicolumn{6}{|l|}{ NNRTI } \\
\hline Any NNRTI & $30(23)$ & $94(81)$ & $131(87)$ & $59(94)$ & $36(95)$ \\
\hline K103N & $13(10)$ & $43(37)$ & $65(43)$ & $29(46)$ & 15 (39) \\
\hline Y188LCH & $1(1)$ & $10(8.6)$ & $14(9)$ & $4(6)$ & $1(3)$ \\
\hline G190 ASEQ & 0 & $23(20)$ & $39(26)$ & $23(37)$ & $14(37)$ \\
\hline $\begin{array}{l}\text { Pan-NNRTI: L100I, } \\
\text { K101EP, Y181, Y188L, } \\
\text { G190, M230L }\end{array}$ & $12(9)$ & $67(58)$ & $96(64)$ & $48(76)$ & 30 (79) \\
\hline Any NRTI + NNRTI & $7(5)$ & $74(64)$ & $112(74)$ & $54(86)$ & $33(87)$ \\
\hline Any NRTI or NNRTI & $36(27)$ & $95(82)$ & 134 (89) & $59(94)$ & 36 (95) \\
\hline
\end{tabular}

Nucleoside reverse transcriptase inhibitor (NRTI), non-nucleoside reverse transcriptase inhibitor (NNRTI), thymidine analog mutations (TAMs)

Median time (IQR) from VF to LG: 202 days (IQR: 139-461)

$\mathrm{VF} \geq 9 \mathrm{mo}$ : subset of patients followed on failing first line ART regimen for 9 months or longer

$\mathrm{VF} \geq 15 \mathrm{mo}$ : subset of patients followed on failing first line ART regimen for 15 months or longer 